\title{
Comments on "Spatial hidden Markov chain models for estimation of petroleum reservoir categorical variables"
}

\author{
Weidong $\mathrm{Li}^{1}$ • Chuanrong Zhang ${ }^{1}$
}

Received: 21 June 2016/Accepted: 25 December 2016/Published online: 31 January 2017

(C) The Author(s) 2017. This article is published with open access at Springerlink.com

Huang et al. (2016a) claimed that they proposed a "spatial hidden Markov chain (SHMC) model" based on the Markov chain random field (MCRF) theory proposed by $\mathrm{Li}$ (2007). However, we found that their so-called SHMC model is exactly the same as the colocated MCRF cosimulation (CoMCRF) model with one auxiliary categorical data set. The coMCRF model was first presented on GIScience 2012 conference ( $\mathrm{Li}$ and Zhang 2012) and then presented on GeoComputation 2013 conference ( $\mathrm{Li}$ and Zhang 2013a). It was mentioned in $\mathrm{Li}$ and Zhang (2013b), described and used in the research article Li et al. (2013), and then further described and used in the research article Li et al. (2015). However, Huang et al. (2016a) seemingly ignored the coMCRF model in all of these published online materials and journal articles. Obviously, Huang et al. reinterpreted the coMCRF model and claimed that they proposed a SHMC model in Huang et al. (2016a). In addition, Huang et al. (2016a) performed an inaccurate case study to support their claim for article publication by apparently using computer programs for other models; they made many inaccurate statements about geostatistics, Markov chain models, and Markov random fields, which are misleading. This is not their first problematic article related to the MCRF model. It is our obligation to clarify the misunderstandings and point out the mistakes.

This comment refers to the article available at doi:10.1007/s13202016-0251-9.

Chuanrong Zhang

chuanrong.zhang@uconn.edu

1 Department of Geography, University of Connecticut, Storrs, CT 06268, USA

\section{Introduction}

In "Introduction" section, Huang et al. (2016a) stated "Spatial Markov chain (SMC) models have also been widely adopted in petroleum reservoir to characterize the spatial heterogeneity of categorical variables through the conditional probabilities (transition probabilities) from different directions (Carle and Fogg 1997; Weissmann and Fogg 1999)". However, Carle and Fogg (1997) introduced continuous-lag Markov chain models, which were actually a joint modelling method of spatial transition probabilities for providing transition probability parameters to transition probability-based indicator geostatistics (TPROGS) (Carle and Fogg 1996). Weissmann and Fogg (1999) was focused on hydrofacies modelling using TPROGS, in which what SMC models were adopted by them? Then, Huang et al. (2016a) grouped both the coupled Markov chain (CMC) model proposed by Elfeki and Dekking (2001) and the MCRF model proposed by Li (2007) into SMC models. Thus, they regarded the CMC model, the MCRF model, and the TPROGS model all as SMC models, no matter whether these models are kriging models or nonlinear Markov chain models. We don't think it is proper to call all of these three models "SMC models", although they all use transition probabilities, while neither Carle and Fogg nor Elfeki and Dekking call their model a "spatial Markov chain" model. Indeed, Li (2007) used the term "spatial Markov chain" to refer to the Markov chain in a specific MCRF and also used the name of "spatial Markov chain model" for specific MCRF models with different numbers of nearest neighbours and spatial dimensions (i.e. one to six nearest neighbours in cardinal directions, and one to three dimensions). However, considering that this term was not sufficiently unique to represent "single-chain-based multidimensional Markov chain model" (Li and Zhang 2008),

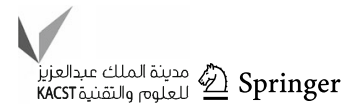


Li (2007) and our later papers used the name of "Markov chain random field" to differentiate the new Markov chain model from other Markov chain models for spatial data. Once the name of a model was decided by the model proposer(s), it is not respectful for others to rename it without sufficient reasons and scientific progress.

Huang et al. (2016a) stated "Most traditional geostatistical models, like Markov chain random field (MCRF), use well data only and make prediction based on SMC, which results in a relatively low prediction accuracy (Huang et al. 2016b)". This is not true! First, traditional geostatistical models such as cokriging and indicator cokriging that make use of auxiliary data have existed for decades. The MCRF model is not a traditional geostatistical model. It even has not been well developed into a software system for release. Second, Huang et al. (2016b) did not use a proper MCRF model to make prediction, but just played a trick to mislead readers by mistakenly claiming that they derived the MCRF model from the conventional Markov random field model. Third, coMCRF model, which may use one or multiple auxiliary data sets, has already been proposed ( $\mathrm{Li}$ et al. 2013, 2015). Huang et al. (2016a) stated "Huang et al. (2016c) introduced a beta-transformed Bayesian updating model to boost the classification accuracy of category random field". We don't think that Huang et al. (2016c) used the proper simulation algorithms and computer programs to perform simulations, except that they just wanted to quickly claim new geostatistical models even without understanding the differences between spatial models and non-spatial models. They further stated: "Auxiliary information, however, has not been taken into consideration". If this was true, then what had Li et al. (2013) and Li et al. (2015) done? Didn't these two papers present the coMCRF model for incorporating auxiliary information into the MCRF model? Didn't these two papers conduct cosimulations for soil class map updating and land cover post-classification, respectively? After the proposition of the MCRF model as a geostatistical model, its extension for collaborative modelling is a natural thing. In addition, both the MCRF model and the coMCRF model are not limited to two dimensions theoretically.

\section{Model}

The second section of the paper is "Review of Markov models". In this section, both Markov mesh models and spatial Markov chain models were reviewed. Let's see how Huang et al. (2016a) made their reviews.

For Markov mesh models, Huang et al. (2016a) wrote their model formula as a conditional probability

$\operatorname{Pr}\left(f_{s} \mid f_{\eta_{s}}\right)=\operatorname{Pr}\left(f_{s} \mid f_{s-1}, f_{s_{1}}, f_{s_{2}}, \ldots, f_{s_{l}}\right)$, and stated that "where $s_{1}, s_{2}, \ldots, s_{l}$ is its nearest known locations of current cell $s$ in different directions; $s-1$ is always the start cell of the Markov chain to the unknown cell $s$, which is to be estimated". It is widely known that Markov mesh models are unilateral lattice models. Here Huang et al. (2016a) suddenly redefined them on surrounding nearest known locations as a geostatistical model (i.e. a model defined on spatial sample data). The purpose of their making such a misleading definition appeared later at the end of this section, where Huang et al. (2016a) stated that "A spatial Markov chain model may be viewed as a special case of Markov mesh models, whereas a Markov mesh model is an extension of spatial Markov chain models, called a generalized spatial Markov chain model". Such a statement is absolutely irrational. Where did Huang et al. find such knowledge? Markov mesh models have existed for half century since 1960s (Abend et al. 1965). How could they generalize the MCRF model proposed in 2007? Here Huang et al. (2016a) turned so-called SMC models, including the CMC model of Elfeki and Dekking (2001), the MCRF model of Li (2007), and the TPROGS method according to their classification, all into special cases of Markov mesh models. Huang et al. (2016a) "generalized" all of these non-unilateral models into unilateral lattice models. Couldn't Huang et al. understand the differences between unilateral models and non-unilateral models and the differences between lattice models and geostatistical models?

For spatial Markov chain models, Huang et al. (2016a) first presented a "generalized form" of the CMC model

$\operatorname{Pr}\left(f_{s} \mid f_{s-1}, f_{s_{1}}, \ldots, f_{s_{l}}\right)=\frac{p_{f_{s-1} f_{s}} p_{f_{s_{1}} f_{s}}^{1} \ldots p_{f_{s} f_{s}}^{l}}{\sum_{f_{s}} p_{f_{s-1} f_{s}} p_{f_{s_{1}} f_{s}}^{1} \ldots p_{f_{s} f_{s}}^{l}}$

by mimicking the generalized form of the MCRF model simplified using the conditional independence assumption. While the CMC model of Elfeki and Dekking (2001) is composed of two 1-D Markov chains, Huang et al. (2016a) made it composed of $l+1$ 1-D Markov chains. What scientific problem did Huang et al. (2016a) attempt to solve by writing the CMC model like this? Then, Huang et al. (2016a) stated that "Using the conditional independence assumption, $\mathrm{Li}$ (2007) gives the general expression of the conditional probability formula in Eq. (1) at any location $s$ as"

$\operatorname{Pr}\left(f_{s} \mid f_{s-1}, f_{s_{1}}, \ldots, f_{s_{l}}\right)=\frac{p_{f_{s-1} f_{s}} p_{f_{s_{s}}}^{1} \ldots p_{f_{s_{s}}}^{l}}{\sum_{f_{s}} p_{f_{s}}}$

How simple is it? Could Huang et al. use the conditional independence assumption to get such a model without the derivation process of using Bayes' theorem for factorizing the spatial conditional probability distribution function 
over a neighbourhood? How could one use such a model to conduct spatial simulation directly conditional on sample data without first defining the conditional probability function on sparse sample data?

The third section of the paper is "Spatial hidden Markov chain model". Huang et al. (2016a) stated "The SHMC is an extension of SMC". Which SMC? The CMC model or the TPROGS model? They stated "A spatial Markov chain $\ldots$ is characterized by its states and conditional probabilities through Eq. (2) or (3)...”. Thus, both the CMC model and the MCRF model were included, but the TPROGS model was not. They defined a local conditional probability distribution for their SHMC model as

$\operatorname{Pr}\left(f_{s} \mid f_{s-1}, f_{s_{1}}, \ldots, f_{s_{l}}, w_{s}\right)=\frac{\operatorname{Pr}\left(w_{s} \mid f_{s}\right) \operatorname{Pr}\left(f_{s} \mid f_{s-1}, f_{s_{1}}, \ldots, f_{s_{l}}\right)}{\sum_{f_{s}} \operatorname{Pr}\left(w_{s} \mid f_{s}\right) \operatorname{Pr}\left(f_{s} \mid f_{s-1}, f_{s_{1}}, \ldots, f_{s_{l}}\right)}$

But when they defined their prior conditional probabilities, they used the formula

$\operatorname{Pr}\left(f_{s} \mid f_{s-1}, f_{s_{1}}, \ldots, f_{s_{l}}\right)=\frac{p_{f_{s-1} f_{s}} p_{f_{s} f_{s_{1}}}^{(1)} \ldots p_{f_{s} f_{s_{l}}}^{(l)}}{\sum_{f_{s}} p_{f_{s-1} f_{s}} p_{f_{s} f_{s_{1}}}^{(1)} \ldots p_{f_{s} f_{s_{l}}}^{(l)}}$,

which is exactly the simplified MCRF model based on the conditional independence assumption provided in $\mathrm{Li}$ (2007), the same as above Eq. (3). Both a generalized MCRF model equation and specific MCRF models with nearest data only in cardinal directions were provided in $\mathrm{Li}$ (2007, p. 327-329, see "MCRF-based SMC models"). Note that the superscripts used in the MCRF model for directions were unnecessary because the subscript labels were already sufficient; we, therefore, didn't use the superscripts anymore in MCRF model equations in our later publications after 2009.

In the above Eq. (4), their $\operatorname{Pr}\left(w_{s} \mid f_{s}\right)$, that is, their emission probability, was called cross-field transition probability in $\mathrm{Li}$ et al. $(2013,2015)$. Because the coMCRF model may incorporate multiple auxiliary data sets, it may have multiple cross-field transition probability terms, which represent the cross-field relationships between the primary categorical field and multiple different auxiliary fields. Here by combining the above Eqs. (4) and (5) and using a cross-field transition probability (or their emission probability) expression $b_{f_{s} w_{s}}$ for the conditional probability $\operatorname{Pr}\left(w_{s} \mid f_{s}\right)$, the complete form of their so-called SHMC model is

$\operatorname{Pr}\left(f_{s} \mid f_{s-1}, f_{s_{1}}, \ldots, f_{s_{l}}, w_{s}\right)=\frac{b_{f_{s} w_{s}} p_{f_{s-1} f_{s}} p_{f_{s}} f_{s_{1}} \ldots p_{f_{s} f_{s_{l}}}}{\sum_{f_{s}}\left[b_{f_{s} w_{s}} p_{f_{s-1} f_{s}} p_{f_{s}} f_{s_{1}} \ldots p_{f_{s} f_{s_{l}}}\right]}$.

One can see that their SHMC model [i.e. Eq. (6)] is exactly the same as the colocated coMCRF model with one auxiliary data set presented in Li et al. (2013) and Li et al. (2015). For the sake of clarity, the colocated coMCRF model with one auxiliary data set in $\mathrm{Li}$ et al. (2013) is copied here:

$$
\begin{aligned}
p & {\left[i_{0}\left(\mathbf{u}_{0}\right) \mid i_{1}\left(\mathbf{u}_{1}\right), \ldots, i_{m}\left(\mathbf{u}_{m}\right) ; r_{0}\left(\mathbf{u}_{0}\right)\right] } \\
& =\frac{b_{i_{0} r_{0}} p_{i_{1} i_{0}}\left(\mathbf{h}_{10}\right) \prod_{g=2}^{m} p_{i_{0} i_{g}}\left(\mathbf{h}_{0 g}\right)}{\sum_{f_{0}=1}^{n}\left[b_{f_{0} r_{0}} p_{i_{1} f_{0}}\left(\mathbf{h}_{10}\right) \prod_{g=2}^{m} p_{f_{0} i_{g}}\left(\mathbf{h}_{0 g}\right)\right]} .
\end{aligned}
$$

The colocated coMCRF model with one auxiliary data set used in Li et al. (2015) is also copied here:

$$
\begin{aligned}
p & {\left[i_{0}\left(\mathbf{u}_{0}\right) \mid i_{1}\left(\mathbf{u}_{1}\right), \ldots, i_{m}\left(\mathbf{u}_{m}\right) ; r_{0}\left(\mathbf{u}_{0}\right)\right] } \\
& =\frac{q_{i_{0} r_{0}} p_{i_{1} i_{0}}\left(\mathbf{h}_{10}\right) \prod_{g=2}^{m} p_{i_{0} i_{g}}\left(\mathbf{h}_{0 g}\right)}{\sum_{f_{0}=1}^{n}\left[q_{f_{0} r_{0}} p_{i_{1} f_{0}}\left(\mathbf{h}_{10}\right) \prod_{g=2}^{m} p_{f_{0} i_{g}}\left(\mathbf{h}_{0 g}\right)\right]} .
\end{aligned}
$$

Please note the differences in symbols: the neighbourhood size for the MCRF model was denoted as $m$ rather than $l+1$, and the cross-field transition probability term was denoted as $b_{i_{0} r_{0}}$ and $q_{i_{0} r_{0}}$ in Li et al. (2013) and Li et al. (2015), respectively. Readers who have interests in the MCRF approach (i.e. Markov chain geostatistics) may read Li (2007), Li and Zhang (2008), and Li et al. (2013, 2015) for details.

Now it is clear that both claiming the CMC model, the MCRF model, and the TPROGS model all as SMC models and messing up these models with Markov mesh models through wrong statements were just tricks played by Huang ret al. (2016a).

\section{Case study}

The fourth section of Huang et al. (2016a) is "Case study". Let's see how they did their simulation. They estimated experimental transiograms from 509 data points and fitted all of them with exponential models. They did not use our transiogram joint modelling methods (Li and Zhang 2010), nor use the transition rate method for continuous-lag Markov chain models (also a transition probability joint modelling method) suggested by Carle and Fogg (1997). How did they guarantee their transiogram models (see Huang et al. 2016a, figure 7) sum to unity in each row? Without satisfying this condition, how could they properly implement their simulations using transiogram models?

What are unbelievable are their simulated results provided in the figure 10 in Huang et al. (2016a) (see below Fig. 1). Huang et al. (2016a) claimed that these results were generated by the "SMC" defined by Eq. (5), which is the simplified MCRF model based on the conditional independence assumption. These results, however, have no way to be the results produced by the simplified MCRF model. The authors also pointed out some problems about 


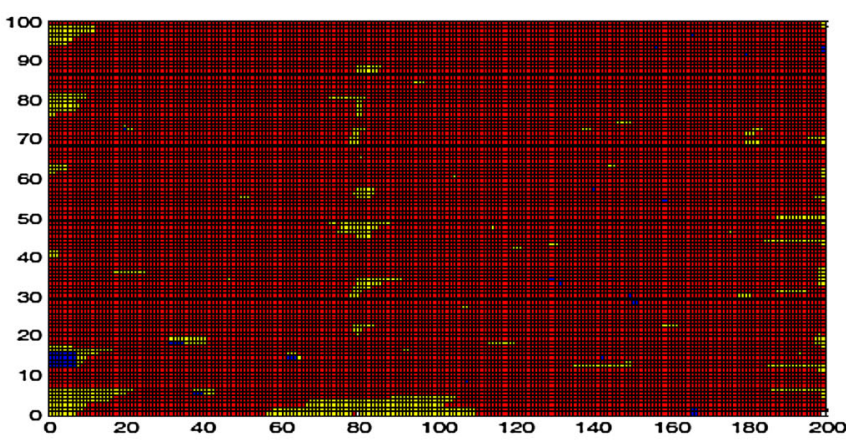

(a)

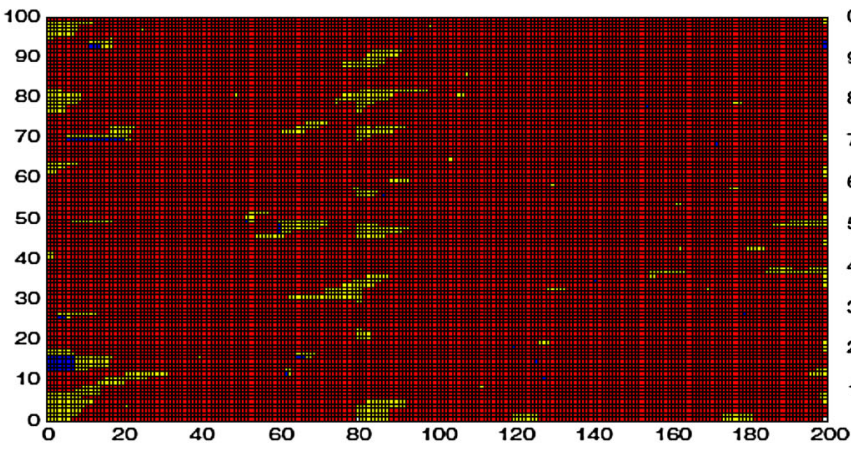

(c)

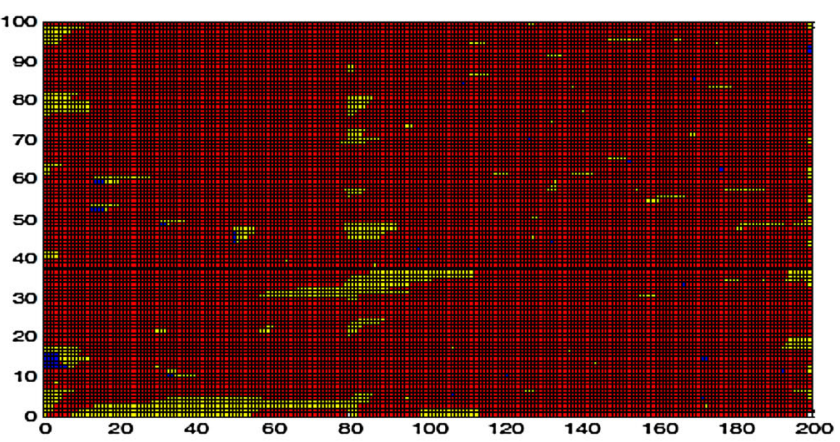

(b)

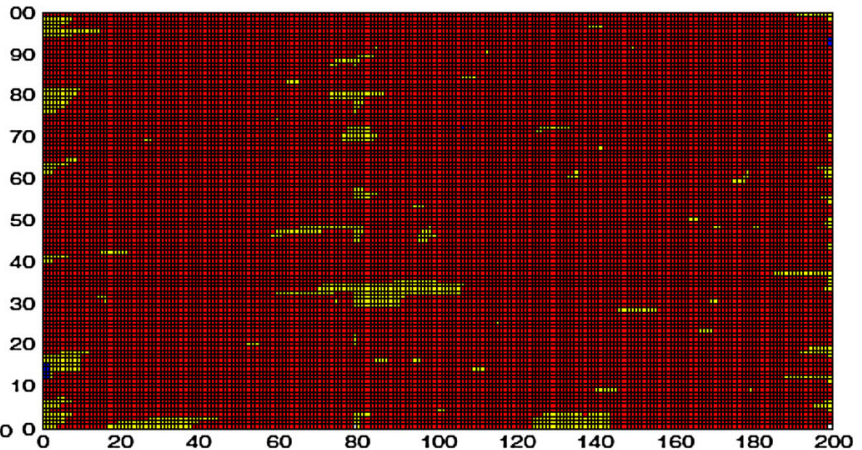

(d)

Fig. 1 Four stochastic simulation results based on three wells using SMC (from Huang et al. 2016a, figure 10)

the results, as they stated "It is obvious that conditional data have played a role in controlling the distribution of lithofacies near the wells. However, the further counterparts are fragmented and random in the grid". We are afraid that the true problem is not fragmentation or randomness. The true problem with the results should be that small classes were strongly underestimated. These results are very similar to the results generated by the unconditional CMC model (see Elfeki and Dekking 2001). This means that Huang et al. (2016a) used the unconditional CMC model to conduct the simulations. It is not surprising because the CMC model used in case studies was always unconditional (to sample data ahead) in their earlier publications (see Liang et al. 2014, Huang et al. 2016b).

\section{Conclusions}

Huang et al. (2016a) claimed "We have presented a SHMC model for geological facies modelling. This combines spatial Markov chain theory and Bayes estimation. We have adopted the specification of earlier published hidden Markov models. SHMC is based on neighbourhood and cliques and has a solid theoretical foundation". These claims are wrong. What Huang et al. (2016a) presented and claimed as a new model is exactly the colocated coMCRF model with one auxiliary data set proposed and published by $\mathrm{Li}$ and his coauthors. What they did was just ignoring the existence of the coMCRF model and giving it a different interpretation. They did not solve any scientific issues. More surprisingly, in their case study, Huang et al. (2016a) actually implemented the CMC model without conditioning to borehole data ahead, but claimed they implemented the MCRF model.

Apparently by misrepresentation, Huang and his coauthors not only claimed the coMCRF model as their new model but also caused some confusion on the whole MCRF approach. According to their logic, cokriging could be called "hidden kriging" and reproposed as a new model, and indicator cokriging could be called "hidden indicator kriging" and proposed as a new model. In summary, Huang et al. (2016a) have mislead their readers. It's our responsibility to clarify their errors and imporper claims.

Open Access This article is distributed under the terms of the Creative Commons Attribution 4.0 International License (http:// creativecommons.org/licenses/by/4.0/), which permits unrestricted use, distribution, and reproduction in any medium, provided you give appropriate credit to the original author(s) and the source, provide a link to the Creative Commons license, and indicate if changes were made.

\section{References}

Abend K, Harley TJ, Kanal LN (1965) Classification of binary random patterns. IEEE Trans Inf Theory 11:538-544 
Carle SF, Fogg GE (1996) Transition probability-based indicator geostatistics. Math Geol 28(4):453-477

Carle SF, Fogg GE (1997) Modeling spatial variability with one and multidimensional continuous-lag Markov chains. Math Geol 29(7):891-918

Elfeki A, Dekking M (2001) A Markov chain model for subsurface characterization: theory and applications. Math Geol 33(5):569-589

Huang X, Li J, Liang Y, Wang Z, Guo J, Jiao P (2016a) Spatial hidden Markov chain models for estimation of petroleum reservoir categorical variables. J Petrol Explor Prod Technol. doi:10.1007/s13202-016-0251-9

Huang X, Wang Z, Guo J (2016b) Theoretical generalization of Markov chain random field from potential function perspective. J Cent South Univ 23(1):189-200

Huang X, Wang Z, Guo J (2016c) Prediction of categorical spatial data via Bayesian updating. Int J Geogr Inf Sci 30(7):1426-1449

Li W (2007) Markov chain random fields for estimation of categorical variables. Math Geol 39(3):321-335

Li W, Zhang C (2008) A single-chain-based multidimensional Markov chain model for subsurface characterization. Environ Ecol Stat 15:157-174

Li W, Zhang C (2010) Linear interpolation and joint model fitting of experimental transiograms for Markov chain simulation of categorical spatial variables. Int J Geogr Inf Sci 24(6):821-839
Li W, Zhang C (2012) A Bayesian Markov chain approach for land use classification based on expert interpretation and auxiliary data. GIScience 2012 Extended Abstracts, Sept. 19-21, Columbus, Ohio. http://www.giscience.org/past/2012/proceedings/ abstracts/giscience2012_paper_137.pdf

Li W, Zhang C (2013a) Updating categorical soil map with limited survey data by Bayesian Markov chain co-simulation. GeoComputation 2013 Extended Abstracts, May 23-25, Wuhan University, China. http://www.geocomputation.org/2013/papers/55.pdf

Li W, Zhang C (2013b) Some further clarification on Markov chain random fields and transiograms. Inter $\mathrm{J}$ Geogr Inf Sci 27(3):423-430

Li W, Zhang C, Dey DK, Willig MR (2013) Updating categorical soil maps using limited survey data by Bayesian Markov chain cosimulation. Sci World J. doi:10.1155/2013/587284

Li W, Zhang C, Willig MR, Dey DK, Wang G, You L (2015) Bayesian Markov chain random field cosimulation for improving land cover classification accuracy. Math Geosci 47(2):123-148

Liang YR, Wang ZZ, Guo JH (2014) Reservoir lithology stochastic simulation based on Markov random fields. J Cent South Univ 21(9):3610-3616

Weissmann GS, Fogg GE (1999) Multi-scale alluvial fan heterogeneity modeled with transition probability geostatistics in a sequence stratigraphic framework. J Hydrol 226(1):48-65 